\title{
Multi-nodule of large airway: tracheobronchopathia osteochondroplastica
}

\author{
Dandan Li ${ }^{1}$, Faguang Jin ${ }^{1}$, Yandong Nan ${ }^{1}$, Hua Jiang ${ }^{1}$, Qiao Liu ${ }^{2}$, Hongang Liu ${ }^{3}$, Tao Xin ${ }^{1}$ \\ ${ }^{1}$ Department of Respiratory and Critical Care Medicine, The Second Affiliated Hospital of Air Force Medical University, Xi'an, China; ${ }^{2}$ Department \\ of Pathology, The Second Affiliated Hospital of Air Force Medical University, Xi'an, China; ${ }^{3}$ Department of Thoracic Surgery, The Second Affiliated \\ Hospital of Air Force Medical University, Xi'an, China \\ Contributions: (I) Conception and design: T Xin, Y Nan; (II) Administrative support: F Jin; (III) Provision of study materials or patients: H Jiang; (IV) \\ Collection and assembly of data: D Li, Q Liu, H Liu; (V) Data analysis and interpretation: D Li, T Xin; (VI) Manuscript writing: All authors; (VII) \\ Final approval of manuscript: All authors. \\ Correspondence to: Tao Xin. Department of Respiratory and Critical Care Medicine, The Second Affiliated Hospital of Air Force Medical University, \\ No. 1, Xinsi Road, Baqiao District, Xi’an 710000, China. Email: tdhxnk@163.com.
}

Background Tracheobronchopathia osteochondroplastica (TO) is a benign rare disease characterized by multiple tracheobronchial nodules, which has not been clearly illuminated and there are no standard guidelines for TO management. The aim of this study was to clarify the clinical features and treatment outcomes of TO and provide basis for clinical diagnosis and treatment.

Methods: A total of 32,215 patients taken bronchoscopy were retrospectively reviewed to select patients diagnosed with TO. Clinical characteristics, bronchoscopic manifestations, CT images, histopathological features, treatments and clinical course were investigated.

Results: 13 males and 6 females were identified as TO. The average age was $47.42 \pm 12.12$ years. The incidence rate of TO in our study by bronchoscopy was $0.06 \%$. The most frequent manifestation was chronic cough. Based on bronchoscopic manifestations, patients were categorized as three groups: the largest proportion was group of Stage II (10/19), followed by stage III (5/19) and stage I (4/19). The positive rate of CT in stage I, stage II and stage III was $0 \%, 50 \%, 100 \%$, respectively. Histopathological analysis presented inflammatory exudation, squamous metaplasia, submucosal cartilaginous and osseous deposition. Bronchoscopic intervention and inhaled corticosteroids were administered to subjects in stage II and III, which improved their life qualities to some degree.

Conclusions: Not only multi-disciplinary cooperation of clinical, endoscopic and histological assessment, but also awareness are crucial to TO diagnosis, especially in early stage of TO, which was difficult to identify. The stage of TO based on bronchoscopic visualization might be applied to guide the choice of clinical treatment strategy.

Keywords: Airway disease; bronchoscopic treatment; chronic cough; rare diseases; tracheobronchopathia osteochondroplastica (TO)

Submitted Feb 04, 2020. Accepted for publication Aug 21, 2020.

doi: $10.21037 /$ apm-20-316

View this article at: http://dx.doi.org/10.21037/apm-20-316

\section{Introduction}

Tracheobronchopathia osteochondroplastica (TO) is a rare benign disease with a stable or indolent course, which is characterized by the formation of multiple cartilaginous or osseous submucosal nodules that originate from the cartilaginous rings of the airway projecting into the tracheobronchial lumen, spare the posterior membranous wall, causing hoarseness, persistent and/or productive cough, hemoptyses, dyspnea and some other symptoms (1). The misdiagnosis and missed diagnosis are very common 
for its variable and non-specific clinical presentation, especially in the early stage of the disease, which can be asymptomatic. TO is usually discovered by accident. The diagnosis rate has been increased significantly with the development of CT, bronchoscopy techniques, and improved awareness. However, there are no standard guidelines for TO management, and most studies about TO were case reports or small series. Thus, the present study aimed to enhance the knowledge and describe the diagnosis and treatment strategy of TO by analyzing the clinical data of 19 patients with TO retrospectively.

We present the following article in accordance with the STROBE reporting checklist (available at http://dx.doi. org/10.21037/apm-20-316).

\section{Methods}

\section{Study design and data collection}

The trial was conducted in accordance with the Declaration of Helsinki (as revised in 2013). This study was approved by the ethics committee of The Second Affiliated Hospital of Air Force Medical University (No.2013027). All of the subjects signed the informed consent form before they underwent bronchoscopic inspection. Because of the retrospective nature of the study, informed consent on human research was not obtained from the participants. Waiver of consent to use the patient records was granted by the Institutional Review Board, and analysis of this study was carried out after anonymization of the patients' data.

A total of 32,215 subjects had taken bronchoscopic examination between January 2014 and December 2018 in the Department of Respiratory and Critical Care Medicine of The Second Affiliated Hospital of Air Force Medical University. 19 patients were identified as TO with histopathologic findings and 2 pulmonary physicians who had more than 5 years of experience in bronchoscopy. Medical records of these patients including demographics, clinical presentations, bronchoscopic and histopathologic features, managements and outcomes were retrospectively analyzed. All patients were followed up for 2 years.

According to the classification criteria developed in a large Chinese cohort study by the characteristic bronchoscopic image (2), TO was divided into three stages: The bronchoscopic visualization of stage I (early stage, also a mild grade) exhibits mucosa hyperemia edema and yellow-whitish soft lesions with plaque-like infiltrations covering the lumen mucosa scatteredly; stage II (middle stage, moderate grade) reveals cartilaginous nodules and sessile spicules protruding into the lumen dispersedly or diffusely with a cobblestone or stalactite appearance; stage III demonstrates airway narrowness even obstruction with a deformed and rigid tracheal wall.

\section{Statistical analysis}

Continuous variables are described by using mean \pm SD . Categorical variables are described as number (percentage). Categorical variables $(<5)$ were analyzed with Fisher's exact test and continuous variables were assessed by Student $t$-test. The significance level of statistical tests was set at $\mathrm{P}<0.05$. All statistical analyses were conducted using $\mathrm{R}$ version 3.5.1.

\section{Results}

Among the 32,215 patients, 19 (male, 13; overall rate, $0.06 \%$ ) were diagnosed as TO. The age ranged from 16 to 65 years old (the average age, $47.42 \pm 12.12 \mathrm{y}$; male $v$ s. female, $\mathrm{P}=0.52$ ). Among the 19 patients, 6 had a history of chronic, recurrent bronchitis or pneumonia. One had been misinterpreted as asthma. Two subjects were suffered from cancer concomitantly (one in lung adenocarcinoma, and the other in postoperative thyroid gland cancer). Two had esophageal disease (One in gastritis and reflux esophagitis, and the other in leucoplakia of esophageal mucosa). Two had been previously diagnosed with infectious disease (one in chronic hepatitis $\mathrm{B}$, and the other in tuberculosis). One had porokeratosis and one had gout.

The most frequent manifestation was chronic cough $(15 / 19)$. Other clinical complaint included sputum production (7/19), dyspnea on exertion (12/19), hemoptysis (4/19), chest tightness and pain (4/19), continuous or intermittent fever (6/19), dryness of the throat (1/19), hoarseness (1/19). What is more, 2 patients were asymptomatic. Abnormal findings on chest auscultation such as wheezing and stridor were found in 3 patients.

In the light of the bronchoscopic manifestations, patients were categorized as three groups as shown in Figure 1: the largest proportion in the current research was group of Stage II incorporating 10 subjects, followed by stage III (5 subjects) and stage I (4 subjects), respectively.

Twelve subjects underwent chest CT in our hospital and 1 in other hospital as shown in Figure 1. Submucosal calcifications were present in 8 individuals $(61.54 \%)$, sparing the posterior membranous trachea. Among which $3(23.07 \%)$ showed annular or linear calcification along 

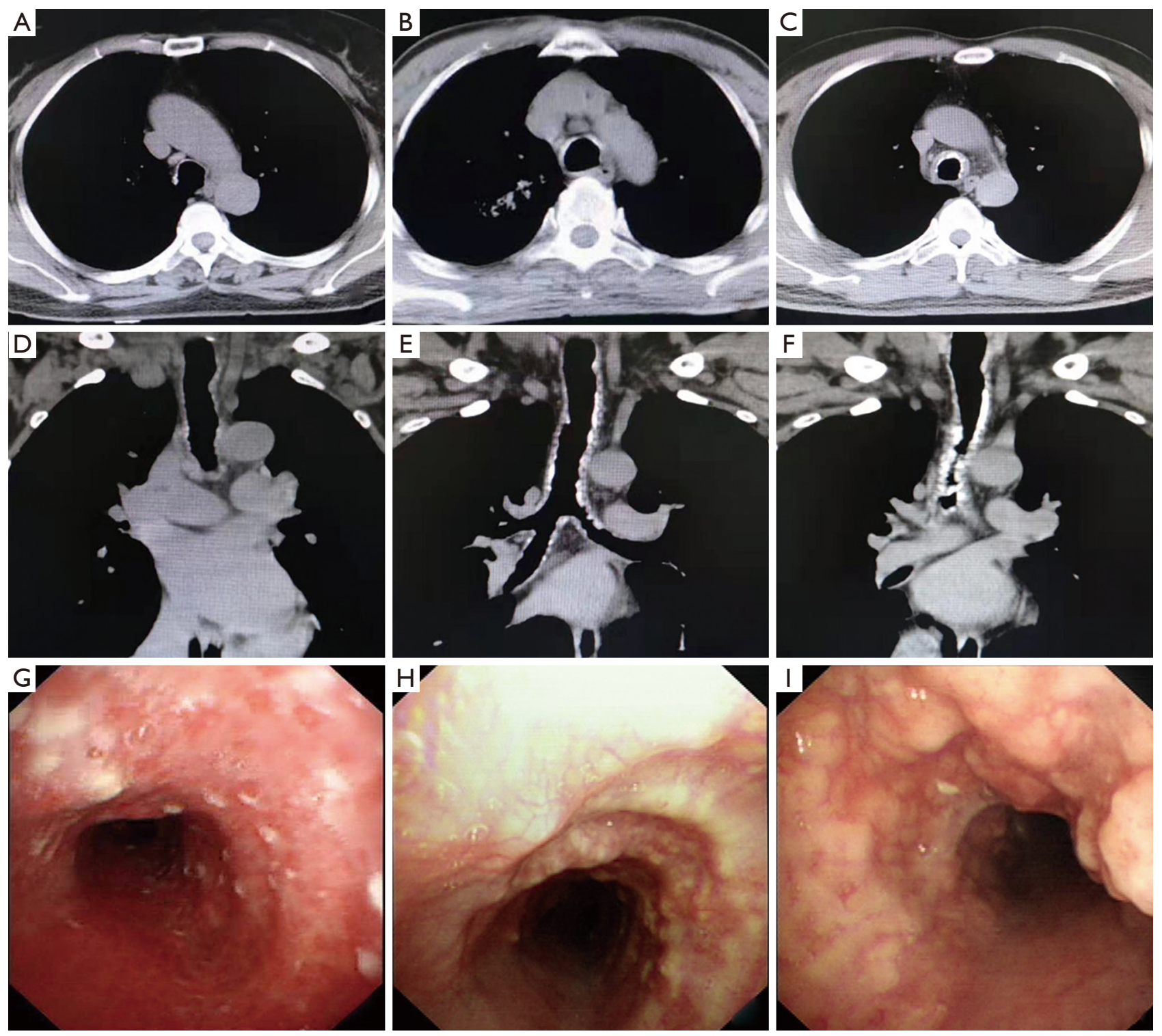

Figure 1 Imaging of TO in different stages. CT scans of TO in stage I (A,D), stage II (B,E), stage III (C,F). Bronchoscopic image of TO in stage I (G), stage II (H), stage III (I). TO, tracheobronchopathia osteochondroplastica.

the inner lining of the tracheobronchial wall, thickening of the tracheobronchial wall, but the tracheobronchial wall remained smooth; 5 (38.46\%) presented with rough tracheobronchial wall and main tracheobronchial stenosis with multiple irregular calcified nodules protruding into the lumen. $1(7.70 \%)$ displayed irregular thickening of the tracheal wall with no calcification and the other 4 (30.77\%) had no abnormal findings in the tracheal wall and lumen. The positive rate of CT in stage I, stage II and stage III was $0,50 \%, 100 \%$, respectively (stage I vs. stage II, $\mathrm{P}=0.22$; stage II vs. stage III, $\mathrm{P}=0.11$; stage I vs. stage III, $\mathrm{P}=0.01$ ).

Histopathological analysis, as shown in Figure 2, presented inflammatory exudation, squamous metaplasia of the ciliated columnar of respiratory epithelium. Cartilaginous and osseous deposited in the underlying submucosa, accompanied by fibrous tissue proliferation, mineralized acellular protein matrix and chronic inflammation cell infiltration.

One subject in stage III with sever obstruction in the tracheal and four in stage II underwent repeated 

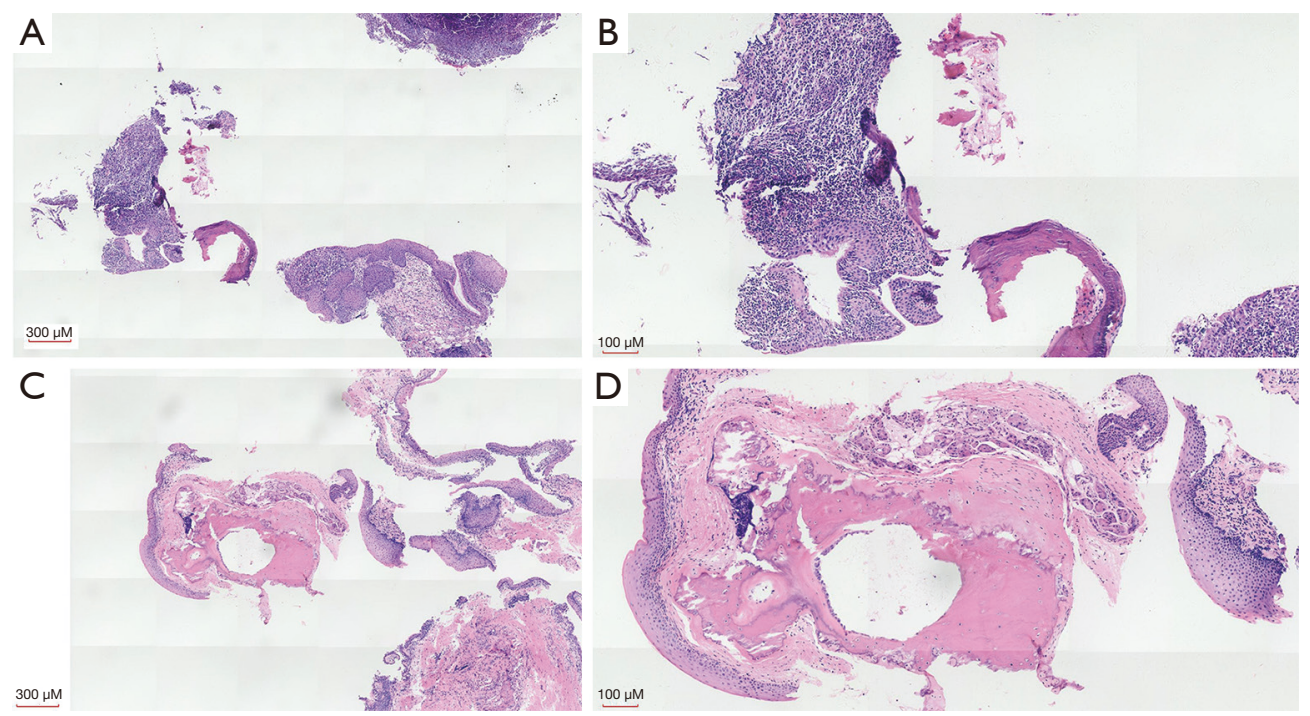

Figure 2 Histopathological findings of TO. Squamous metaplasia of the bronchial mucosa, infiltration of chronic inflammatory cells in the bronchial mucosa, cartilaginous and osseous deposition underneath the respiratory epithelium (bar in A and C: $300 \mu \mathrm{m}$; bar in B and D: $100 \mu \mathrm{m})$. TO, tracheobronchopathia osteochondroplastica.

bronchoscopic intervention such as argon plasma coagulation regularly for 6 to 24 months, among which, the patient in stage III also received balloon dilatation. Additionally, two subjects in stage III and two in stage II were administered inhaled corticosteroids involving Budesonide and Formoterol Fumarate for Inhalation $(160 \mu \mathrm{g} / 4.5 \mu \mathrm{g}$, bid) or Salmetero Xinafoate and Fluticasone propionate Powder for Inhalation $(50 \mu \mathrm{g} / 250 \mu \mathrm{g}$, bid), with duration of treatment varying from 3 to 24 months. Improvement of cough, dyspnea and performance status were observed, however little change was found under bronchoscopy and patient in stage III receiving argon plasma coagulation and balloon dilatation progressed two years later whose bronchoscopic finding displayed bilateral main bronchi stenosis. The lung cancer patient received regular chemotherapy and died one year later, and the other patient took antibiotics, expectorant, antitussive and some other symptomatic treatments showing stable bronchoscopically.

\section{Discussion}

TO was originally described in 1857 by Wilks, who reported autoptic findings of a number of bony plates in a 38-year-old man who died of tuberculosis (3). Ecchondrosis and exostosis proposed by Virchow in 1869 and metaplasia of the elastic tissue by Aschoff in 1910 had been considered as two main theories illuminating the origin and pathogenesis of TO $(4,5)$, which is currently listed under the osteochondrodysplasia category. The incidence rate of TO ranged from 1:400 $(0.25 \%)$ to $3: 1,000$ $(0.30 \%)$ in autopsies and $1: 125(0.80 \%)$ to $1: 10,000(0.01 \%)$ via bronchoscopy (6). The detection rate of this disease in the current study by bronchoscopy was $0.06 \%$, which is consistent with previous reports.

At present, the etiology and pathogenesis of TO have not been fully understood. Coexistence of TO with sarcoidosis, pulmonary adenocarcinoma, breast cancer, esophageal cancer, IgA deficiency and tuberculosis had been reported (7-10). In the current study, we described two TO patients concomitant with lung adenocarcinoma and thyroid gland cancer, respectively. Perhaps there is a similar potential etio-pathogenetic mechanism between TO and malignant diseases, because abnormal bone metabolism is common in cancer especially those with neuroendocrine or endocrine features. Researchers reported TO occurred in recurrent retrosternal goiter, demonstrating an association between TO and thyroid pathology further (11). Adenovirus, coxsackie B virus were also found in TO (12), and in our study we presented TO coexisted with tuberculosis and HBV, supporting chronic infection or inflammation may play a crucial role in the occurrence or exacerbation of TO. Some TO patients were smokers, or suffered from reflux esophagitis or had a long- 
term exposure to dust or irritant gases due to occupational factors, so gastric acid, smoking, dust and irritant gases should also be considered as pathogenic factors. Overall, TO may be attributed to chronic infection or inflammation, cancer, physical and chemical factors, smoking and some other factors.

Chest X-ray is generally normal and there are not typical radiological signs. CT scans showed poor detection rate of TO patients in stage I, which can reveal the characteristic of TO-related tracheobronchial lesions for its sensitivity to calcification. Nevertheless, some radiologist just ignore this sign due to their lack of awareness of TO. So improving awareness is of significant importance for radiologist. Characteristic chest CT scans of TO demonstrate multiple calcified nodules at the anterolateral wall of the trachea with typical sparing of the posterior membranous part (13). Positive percentage of CT in the current study (9/13) was lower than that of Luo's study, which was $87.5 \%$ (28/32). Proportion of cases who didn't undergo CT scans may explain the difference between Luo's study (41/73) and ours (6/19). Additionally, CT image of three-dimensional airway reconstruction should also be taken into consideration. CT virtual bronchoscopy, as a non-invasive technique, could also be applied to detect the characteristic manifestations of TO, which resembles stalactites, or manifests with a rockgarden-like trachea (14).

Bronchoscopy is the gold standard for TO diagnosis. However multi-nodule abnormalities of the tracheobronchus under bronchoscopy was caused by different diseases, 29\% of which were diagnosed as tuberculosis granuloma, and $41.8 \%$ were malignant disease, and $21.8 \%$ were $\mathrm{TO}$, and $3.6 \%$ were sarcoidosis, and $1.8 \%$ lymphoma, $1.8 \%$ fungal infection in An's study (15). Conventional bronchoscopy is insufficient for the detection of subtle mucosal changest and treatment responses. Autofluorescence imaging bronchoscopy (AFI) is a newly developed advanced endoscopic technology identifying subtle mucosal changes with the assistance of different colors, which can detect not only bronchogenic carcinoma in situ but also non-malignant lesions such as metaplasia (16). Japanese research firstly performed AFI in TO, which revealed that the entire circumference of the pathological trachea was in a magenta color while the normal lobar and segmental airways appeared green (17). AFI maybe an appropriate tool to assess the management and follow-up of patients with TO, which can be an important supplementary approach to conventional bronchoscopy. More investigation need to be done about application of AFI in TO.

So far there are no standard guidelines for TO management. Some patients may not need to be overtreated as researchers found that some individuals had experienced a stable and non-progressive course as long as 20 years after diagnosis (18). Long-term clinical observation may be a good choice for patients at stage I. In Zhu's opinion, inhaled corticosteroids might have some impact on patients at Stage I/II (2). Inhaled corticosteroids may be given chronically and regularly especially in those lesions filling with inflammatory cells, because lesions in the airway could not be completely corrected, and airway obstruction could not be reversed. Bronchoscopic intervention, such as argon plasma coagulation, radiofrequency ablation, may be vital for patients in stage II and stage III. Li believed that radiofrequency ablation could be applied to patients with scattered nodules due to its safety and less damage to deep tissue, and argon plasma coagulation may be used to patients with diffuse (widespread) or nodular (concentrated nodules) for its less smoke, less heat conduction and thinner eschar (19). The course and dose of inhaled corticosteroids, the interval of bronchoscopic intervention are still unclear because the limitations of retrospective study with small sample size. Large multicenter prospective study needs to be done to provide new sights about TO.

\section{Conclusions}

In conclusion, $\mathrm{TO}$ is a benign rare disease progressing slowly over years. Awareness is the key for diagnosis and management of TO, which needs multi-disciplinary cooperation of clinical, radiologist, endoscopic and histological assessment as well. Inhaled corticosteroids and bronchoscopic intervention may be two significant methods for TO treatment. The stage of TO based on bronchoscopic visualization might be applied to guide the choice of clinical treatment strategy.

\section{Acknowledgments}

The authors thank the patients and investigators for their participation in this study. The authors thank the colleagues in the Department of Pathology from The First Affiliated Hospital of Air Force Medical University, who gave lots of help about pathology.

Funding: This study was funded by national public health project (201402024). 


\section{Footnote}

Reporting Checklist: The authors have completed the STROBE reporting checklist. Available at http://dx.doi. org/10.21037/apm-20-316

Data Sharing Statement: Available at http://dx.doi. org/10.21037/apm-20-316

Peer Review File: Available at http://dx.doi.org/10.21037/ apm-20-316

Conflicts of Interest: All authors have completed the ICMJE uniform disclosure form (available at http://dx.doi. org/10.21037/apm-20-316). Nobody serves as an unpaid editorial board member (or Section Editor) of Annals of Palliative Medicine. The authors have no conflicts of interest to declare.

Ethical Statement: The authors are accountable for all aspects of the work in ensuring that questions related to the accuracy or integrity of any part of the work are appropriately investigated and resolved. The trial was conducted in accordance with the Declaration of Helsinki (as revised in 2013). This study was approved by the ethics committee of The Second Affiliated Hospital of Air Force Medical University (No.2013027). All of the subjects signed the informed consent form before they underwent bronchoscopic inspection. Because of the retrospective nature of the study, informed consent on human research was not obtained from the participants. Waiver of consent to use the patient records was granted by the Institutional Review Board, and analysis of this study was carried out after anonymization of the patients' data.

Open Access Statement: This is an Open Access article distributed in accordance with the Creative Commons Attribution-NonCommercial-NoDerivs 4.0 International License (CC BY-NC-ND 4.0), which permits the noncommercial replication and distribution of the article with the strict proviso that no changes or edits are made and the original work is properly cited (including links to both the formal publication through the relevant DOI and the license). See: https://creativecommons.org/licenses/by-nc-nd/4.0/.

\section{References}

1. Riva G, Girolami I, Luchini C, et al. Tracheobronchopathia
Osteochondroplastica: A Case Report Illustrating the Importance of Multilevel Workup Clinical, Endoscopic and Histological Assessment in Diagnosis of an Uncommon Disease. Am J Case Rep 2019;20:74-7.

2. Zhu Y, Wu N, Huang HD, et al. A clinical study of tracheobronchopathia osteochondroplastica: findings from a large Chinese cohort. PLoS One 2014;9:e102068.

3. Lundgren R, Stjernberg NL. Tracheobronchopathia osteochondroplastica. A clinical bronchoscopic and spirometric study. Chest 1981;80:706-9.

4. Toth C. [Tracheopathia osteoplastica. A 100-year-old mystery]. Pathologe 2012;33:129-34.

5. Ashley DJ. Bony metaplasia in trachea and bronchi. J Pathol 1970;102:186-8.

6. Prakash UB. Tracheobronchopathia osteochondroplastica. Semin Respir Crit Care Med 2002;23:167-75.

7. Luo T, Zhou H, Meng J. Clinical Characteristics of Tracheobronchopathia Osteochondroplastica. Respir Care 2019;64:196-200.

8. Chrysikos S, Karampitsakos T, Tzouvelekis A, et al. Coexistence of Tracheobronchopathia Osteochondroplastica and Sarcoidosis: Accidental Finding or Something More? J Bronchology Interv Pulmonol 2019;26:e7-8.

9. Dincer HE, Dunitz JM. Tracheobronchopathia osteochondroplastica and selective IgA deficiency. J Bronchology Interv Pulmonol 2012;19:54-6.

10. Sousa M, Silva J, Rodrigues B. Coincident Tuberculosis and Tracheobronchopathia Osteochondroplastica in a Patient. Arch Bronconeumol 2017;53:343.

11. Quaglino F, Mazza E, Navarra M, et al. Tracheobronchopathia osteochondroplastica in recurrent retrosternal goiter. Surgical management. Ann Ital Chir 2017;6:S2239253X17026731.

12. Ge YL, Liu CH, Wang MH, et al. Does Adenovirus and Coxsackie B Virus Infection Play a Role in Tracheobronchopathia Osteochondroplastica (TO). Clin Lab 2018. doi: 10.7754/Clin.Lab.2018.180618.

13. Cai ZG, Quan GM, Zhang HF. Tracheobronchopathia osteochondroplastica: diagnostic value of computed tomography. Am J Med Sci 2015;349:357.

14. Chien YC, Wang HC, Chang YC, et al. Uncommon chronic cough caused by tracheobronchopathia osteochondroplastica. Thorax 2012;67:1021-2.

15. An J, Yang HP, Hu CP, et al. Multinodule abnormalities of the tracheobronchus: bronchoscopy findings and clinical diagnosis. Clin Respir J 2017;11:440-7.

16. Nakanishi K, Ohsaki Y, Kurihara M, et al. Color auto- 
fluorescence from cancer lesions: improved detection of central type lung cancer. Lung Cancer 2007;58:214-9.

17. Ihara H, Tajima K, Yoshikawa H, et al. Autofluorescence imaging bronchoscopy as a novel approach to the management of tracheobronchopathia osteochondroplastica: a case report. J Thorac Dis 2016;8:E1195-8.

Cite this article as: Li D, Jin F, Nan Y, Jiang H, Liu Q, Liu $\mathrm{H}$, Xin T. Multi-nodule of large airway: tracheobronchopathia osteochondroplastica. Ann Palliat Med 2021;10(2):1115-1121. doi: 10.21037/apm-20-316
18. Brandén E. A 20-year follow-up of a case with tracheobronchopathia osteochondroplastica. J Bronchology Interv Pulmonol 2013;20:84-6.

19. Li YY, Hu CP, Yang HZ, et al. The diagnostic value of flexible bronchoscopy in tracheobronchopathia osteochondroplastica. Zhonghua Jie He He Hu Xi Za Zhi 2009;32:489-92. 